\title{
Efficient error models for fault-tolerant architectures and the Pauli twirling approximation
}

\author{
Michael R. Geller and Zhongyuan Zhou \\ Department of Physics and Astronomy, University of Georgia, Athens, Georgia 30602, USA
}

(Dated: March 16, 2018)

\begin{abstract}
The design and optimization of realistic architectures for fault-tolerant quantum computation requires error models that are both reliable and amenable to large-scale classical simulation. Perhaps the simplest and most practical general-purpose method for constructing such an error model is to twirl a given completely positive channel over the Pauli basis, a procedure we refer to as the Pauli twirling approximation (PTA). In this work we test the accuracy of the PTA for a small stabilizer measurement circuit relevant to fault-tolerant quantum computation, in the presence of both intrinsic gate errors and decoherence, and find excellent agreement over a wide range of physical error rates. The combined simplicity and accuracy of the PTA, along with its direct connection to the $\chi$ matrix of process tomography, suggests that it be used as a standard reference point for more refined error model constructions.
\end{abstract}

PACS numbers: 03.67.Lx, 85.25.Cp

\section{PAULI TWIRLING APPROXIMATION}

The principal obstacle to large-scale quantum computation is the introduction errors caused by decoherence, noise, leakage to non-computational states, incorrect implementation of quantum gates, qubit loss, and inaccurate state initialization and measurement. The standard approach for mitigating these errros is to use a faulttolerant error-correction protocol, which enables arbitrarily large computations as long as the strength of the errors are below a threshold value [1 [5] and are not overly correlated in space or time $[6-10]$. The fault-tolerant error threshold is a measure of the robustness of a quantum computing platform and an estimate of its value is one of the most important tasks for practical quantum computer design.

A straightforward approach for calculating logical error rates and associated error thresholds would be to do a full Hilbert space simulation of quantum codes of increasing size, in the presence of decoherence and other errors, but this approach quickly becomes intractable. This difficulty can be circumvented by using special error models that are efficient to simulate classically. The existence of a broad class of these efficient error models, which includes the Pauli and Clifford channels, is provided by the Gottesman-Knill theorem: This theorem states that any quantum circuit consisting only of Clifford-group unitaries and measurement in the Pauli basis can be simulated classically in polynomial time 11,12$]$. The circuits used to implement stabilizer-based error detection and correction - in the absence of any errors - are important examples. However, after including decoherence and intrinsic gate errors, which are required to assess fault-tolerance and calculate error thresholds, the simulations are no longer efficient. By intrinsic we mean an error, such as a unitary qubit rotation by the wrong angle, which does not result from noise or decoherence. The resulting inefficiency of classical simulation is the main motivation for the widely used stochastic approach of modeling nonideal stabilizer circuits by a sequence of one- and two-qubit operations, each of which consists the intended ideal Clifford gate, possibly followed by a unitary "error" randomly drawn from the Pauli or Clifford basis according to some probability distribution. This approach raises several important questions for the design and optimization of realistic quantum computing architectures: What set of error operations - which can also include Pauli-basis measurement - should be allowed? How should the probability distribution over that set be determined? And how reliable is this entire approach?

A first step towards answering these questions was taken recently by Magesan et al. [13] and by Gutiérrez et al. [14]. The focus of Refs. [13] and [14] is similar: Both find the Pauli or Clifford channel closest (according to a chosen norm) to a given target error channel subject to a constraint that the approximation upper-bounds some measure of the error. Here we take a different approach by attempting to directly assess the accuracy of one of the simplest known error model constructions - what can be called the Pauli twirling approximation - when applied to a realistic stabilizer measurement circuit.

The completely positive time evolution of an $N \times N$ density matrix can be represented by

$$
\rho \rightarrow \Lambda(\rho)=\sum_{m} E_{m} \rho E_{m}^{\dagger}
$$

where the $E_{m}$ are $N \times N$ Kraus matrices. The process $\Lambda$ does not need to be trace preserving. Twirling $15-19$ a process $\Lambda$ refers to the pre-multiplication of the input state by an operation $A$, running the original process $\Lambda$, and then post-multiplying by $A^{-1}$; this procedure is then averaged over a set of $K$ operations $\mathcal{A}=\left\{A_{k}\right\}_{k=1}^{K}$. Denoting the twirled process by $\tilde{\Lambda}$, we have

$$
\tilde{\Lambda}(\rho) \equiv \frac{1}{K} \sum_{A \in \mathcal{A}} A^{-1} \Lambda\left(A \rho A^{-1}\right) A .
$$


In this work we will be interested in the case where $\rho$ is an $n$-qubit density matrix, $N=2^{n}, \Lambda$ characterizes the residual error associated with an ideal operation $O$, and $\mathcal{A}$ is the $n$-qubit Pauli basis

$$
\mathcal{A}_{n} \equiv\{I, X, Y, Z\}^{\otimes n}
$$

consisting of the $4^{n}$ distinct tensor products of Pauli matrices $I, X, Y$, and $Z$. Twirling (1) over the Pauli basis (3) results in a new map $\tilde{\Lambda}$ that is diagonal in the Pauli basis [15 19$]$,

$$
\tilde{\Lambda}(\rho)=\sum_{A \in \mathcal{A}_{n}} p_{A} A \rho A^{\dagger}
$$

where the $p_{A}$ are uniquely determined from the Kraus matrices $E_{m}$. If the process (1) is trace preserving, then

$$
\sum_{A \in \mathcal{A}_{n}} p_{A}=1
$$

However there are important error processes, such as those including leakage, where $\sum_{A} p_{A}<1$. The expression (44) shows that the PTA maps every process to an $n$-qubit Pauli channel. In this paper $A$ refers to an element of the Pauli basis $\mathcal{A}_{n}$, with $n \geq 1$, whereas $a$ always represents an element of $\mathcal{A}_{1}$ :

$$
a \in\{I, X, Y, Z\}
$$

Thus we can write every $A \in \mathcal{A}_{n}$ as

$$
A=a_{1} \otimes a_{2} \otimes \cdots \otimes a_{n}, \text { where } a_{i} \in\{I, X, Y, Z\} .
$$

Twirling over the larger Clifford group has also been considered for the purpose of simplifying experimental process tomography [16, 17].

There are several features that make the PTA especially practical for efficient error model construction:

1. The approxmation is generally applicable to any single- or multi-qubit error process. It can be used to model both decoherence and intrinsic gate errors. Examples of each are given in Sec. II]

2. It is straightforward to apply, and, in many cases, leads to simple analytic formulas for the Pauli-error probabilities $p_{A}$.

3. The resulting twirled channel is itself very simple, and contains far fewer terms than the most general classically efficient channel allowed by the Gottesman-Knill theorem. This simplifies the large-scale Monte Carlo simulation of errorcorrected quantum computation with codes such as Autotune [20].

4. The probabilities $p_{A}$ can also be directly measured experimentally without complete process tomography: They are the diagonal elements of the $\chi$ matrix in the Pauli basis [16 19].
An important ingredient of the error model constructions of Refs. [13] and [14] is the bounding property, that the approximations do not overestimate (some measure of) the channel fidelity. However, upper-bounding the channel error does not necessarily upper-bound the logical error probability of a fault-tolerant computation, and in this work we are more concerned with the reliability of simulated logical error rates, especially well below the error threshold. Although the PTA can underestimate the logical error rate, we find that its performance when applied to the four-qubit stabilizer circuit of Sec. III is already suffifciently impressive that there is little room for further improvement - at least for the small system considered here. We will return to this issue in Sec. IV.

The organization of this paper is as follows: In Sec. II we provide two example applications of the PTA. The first is to a somewhat general model of decoherence that includes non-Markovian dephasing, extending the results of Refs. [21] and [22]. The second is to a highfidelity controlled-Z (CZ) gate designed for superconducting qubits. In Sec. III we consider a primitive errorcorrection protocol, that of preserving a pair of qubits in a single given Bell state. We calculate the errorcorrection failure probability in the presence of decoherence and intrinsic gate errors, both exactly and with the PTA, and find remarkable agreement order a wide range of physical error rates. Sec. [V contains our conclusions.

\section{PTA EXAMPLES}

In this section we apply the PTA to typical one-qubit (Sec. IA) and two-qubit (Sec. IB) error channels.

\section{A. Qubit decoherence}

Here we apply the PTA to a model of single-qubit decoherence defined by the map

$$
\begin{aligned}
\rho & =\left(\begin{array}{cc}
1-\rho_{11} & \rho_{01} \\
\rho_{01}^{*} & \rho_{11}
\end{array}\right) \\
& \rightarrow\left(\begin{array}{cc}
1-\rho_{11} e^{-t / T_{1}} & \rho_{01} e^{-t / 2 T_{1}} e^{-\left(t / T_{\phi}\right)^{1+\alpha}} \\
\rho_{01}^{*} e^{-t / 2 T_{1}} e^{-\left(t / T_{\phi}\right)^{1+\alpha}} & \rho_{11} e^{-t / T_{1}}
\end{array}\right),
\end{aligned}
$$

which includes pure dephasing by classical noise with a power spectrum

$$
S(f)=\text { const } \times \frac{1}{f^{\alpha}},
$$

and which is non-Markovian (the time-evolutions do not form a semigroup) if $\alpha \neq 0$. This is described by the channel

$$
\Lambda(\rho)=\sum_{m=1}^{3} E_{m} \rho E_{m}^{\dagger}
$$


with Kraus matrices

$$
\begin{aligned}
E_{1} & =\left(\begin{array}{cc}
1 & 0 \\
0 & \sqrt{1-\gamma-\lambda}
\end{array}\right) \\
& =\frac{1+\sqrt{1-\gamma-\lambda}}{2} I+\frac{1-\sqrt{1-\gamma-\lambda}}{2} Z, \\
E_{2} & =\left(\begin{array}{cc}
0 & \sqrt{\gamma} \\
0 & 0
\end{array}\right)=\frac{\sqrt{\gamma}}{2} X+\frac{i \sqrt{\gamma}}{2} Y, \\
E_{3} & =\left(\begin{array}{cc}
0 & 0 \\
0 & \sqrt{\lambda}
\end{array}\right)=\frac{\sqrt{\lambda}}{2} I-\frac{\sqrt{\lambda}}{2} Z
\end{aligned}
$$

where

$$
\gamma \equiv 1-e^{-t_{\text {step }} / T_{1}}
$$

and

$$
\lambda \equiv e^{-t_{\mathrm{step}} / T_{1}}\left[1-e^{-2\left(t_{\mathrm{step}} / T_{\phi}\right)^{1+\alpha}}\right] .
$$

Here we have assumed that (8) is to be applied to an individual step in a quantum circuit, with time duration $t_{\text {step }}$. The decoherence model (8) reduces to standard $T_{1,2}$ decoherence in the limit $\alpha \rightarrow 0$ and

$$
\frac{1}{T_{\phi}} \rightarrow \frac{1}{T_{2}}-\frac{1}{2 T_{1}} .
$$

To apply the PTA to (8), we expand the Kraus matrices (11) in the single-qubit Pauli basis [see (6)]

$$
E_{m}=\sum_{a \in\{I, X, Y, Z\}} \Gamma_{m a} a,
$$

where the coefficients $\Gamma_{m a}$ are given in Table【. Then (10) is equivalent to

$$
\Lambda(\rho)=\sum_{a, a^{\prime} \in \mathcal{A}_{1}}\left(\sum_{m} \Gamma_{m a} \Gamma_{m a^{\prime}}^{*}\right) a \rho a^{\prime \dagger} .
$$

The PTA replaces (16) by the diagonal Pauli channel

$$
\tilde{\Lambda}(\rho)=\sum_{a \in\{I, X, Y, Z\}} p_{a} a \rho a^{\dagger},
$$

with

$$
p_{a} \equiv \sum_{m}\left|\Gamma_{m a}\right|^{2}
$$

Therefore, in the PTA the single-qubit decoherence model (8) becomes

$$
\begin{aligned}
\rho & \rightarrow\left(1-p_{X}-p_{Y}-p_{Z}\right) \rho \\
& +p_{X} X \rho X+p_{Y} Y \rho Y+p_{Z} Z \rho Z,
\end{aligned}
$$

where

$$
\begin{aligned}
p_{X} & =\frac{\gamma}{4}=\frac{1-e^{-t_{\text {step }} / T_{1}}}{4} \approx \frac{t_{\text {step }}}{4 T_{1}}, \\
p_{Y} & =\frac{\gamma}{4}=\frac{1-e^{-t_{\text {step }} / T_{1}}}{4} \approx \frac{t_{\text {step }}}{4 T_{1}}, \\
p_{Z} & =\frac{1}{2}-\frac{\gamma}{4}-\frac{\sqrt{1-\gamma-\lambda}}{2} \\
& =\frac{1}{2}-\frac{1-e^{-t_{\text {step }} / T_{1}}}{4}-\frac{e^{-t_{\text {step }} / 2 T_{1}} e^{-\left(t_{\text {step }} / T_{\phi}\right)^{1+\alpha}}}{2} \\
& \approx \frac{1}{2}\left(\frac{t_{\text {step }}}{T_{\phi}}\right)^{1+\alpha} \cdot
\end{aligned}
$$

The approximate expressions in (20) apply when $t_{\text {step }} \ll$ $T_{1}, T_{\phi}$.

TABLE I. Kraus matrix coefficients for decoherence.

\begin{tabular}{|c|c|c|c|c|}
\hline$\Gamma_{m a}$ & $a=I$ & $a=X$ & $a=Y$ & $a=Z$ \\
\hline$m=1$ & $\frac{1+\sqrt{1-\gamma-\lambda}}{2}$ & 0 & 0 & $\frac{1-\sqrt{1-\gamma-\lambda}}{2}$ \\
\hline$m=2$ & 0 & $\frac{\sqrt{\gamma}}{2}$ & $\frac{i \sqrt{\gamma}}{2}$ & 0 \\
\hline$m=3$ & $\frac{\sqrt{\lambda}}{2}$ & 0 & 0 & $-\frac{\sqrt{\lambda}}{2}$ \\
\hline
\end{tabular}

The Pauli channel (19) reduces to the depolarization channel $\left(p_{X}=p_{Y}=p_{Z}\right)$ if

$$
\lambda=\gamma(1-\gamma),
$$

which occurs when

$$
T_{\phi}=T_{\phi}^{\mathrm{crit}}
$$

where

$$
T_{\phi}^{\text {crit }} \equiv t_{\text {step }}^{\frac{\alpha}{1+\alpha}}\left(2 T_{1}\right)^{\frac{1}{1+\alpha}} .
$$

Condition (22) generalizes the usual Markovian-limit requirement of $T_{2}=T_{1}$ (or $T_{\phi}=2 T_{1}$ ) and is important because it specifies a crossover in $T_{\phi}$ beyond which phaseflip errors become subdominant to bit-flip errors (dephasing no longer becomes harmful).

The decoherence model (8) is extended to $n>1$ qubits by letting the index $m$ in (1) be the $n$-tuple $\left(m_{1}, m_{2}, \ldots, m_{n}\right)$, with $m_{i} \in\{1,2,3\}$, and

$$
E_{\left(m_{1}, \ldots, m_{n}\right)}=E_{m_{1}} \otimes E_{m_{2}} \otimes \cdots \otimes E_{m_{n}},
$$

where the $E_{m_{i}}$ are the single-qubit Kraus matrices given in (11). Then

$$
\begin{aligned}
\Lambda(\rho) & =\sum_{m_{1}, \ldots, m_{n}} E_{m_{1}} \otimes E_{m_{2}} \otimes \cdots \otimes E_{m_{n}} \rho \\
& \times E_{m_{1}}^{\dagger} \otimes E_{m_{2}}^{\dagger} \otimes \cdots \otimes E_{m_{n}}^{\dagger},
\end{aligned}
$$

where $\rho$ is an $n$-qubit density matrix. Using (15) leads to the twirled multi-qubit decoherence channel

$$
\tilde{\Lambda}(\rho)=\sum_{a_{1}, \ldots, a_{n}} p_{a_{1} a_{2} \cdots a_{n}} a_{1} \otimes a_{2} \otimes \cdots \otimes a_{n} \rho a_{1}^{\dagger} \otimes a_{2}^{\dagger} \otimes \cdots \otimes a_{n}^{\dagger},
$$


where

$$
p_{a_{1} a_{2} \cdots a_{n}}=\prod_{i=1}^{n} p_{a_{i}}
$$

Here the $p_{a_{i}}$ are the single-qubit PTA probabilties given in (20).

\section{B. Nonideal CZ gate}

Next we consider a two-qubit error channel example, that associated with the CZ gate of Strauch et al. [23] for a pair of superconducting qubits or a qubit and resonator bus. The ideal CZ gate in the standard basis $\{|00\rangle,|01\rangle,|10\rangle,|11\rangle\}$ is

$$
\mathrm{CZ} \equiv\left(\begin{array}{cccc}
1 & 0 & 0 & 0 \\
0 & 1 & 0 & 0 \\
0 & 0 & 1 & 0 \\
0 & 0 & 0 & -1
\end{array}\right)
$$

A detailed analysis of the intrinsic errors associated with this gate is given in Ref. [24]. Here we consider a particular subset of error processes dominant in the qubit-qubit case (assuming perfect auxiliary $z$ rotations), and parameterize the nonideal CZ by the unitary

$$
U=\left(\begin{array}{cccc}
1 & 0 & 0 & 0 \\
0 & \sqrt{1-\mathbb{E}_{1}} & \sqrt{\mathbb{E}_{1}} e^{i \phi} & 0 \\
0 & -\sqrt{\mathbb{E}_{1}} e^{-i \phi} & \sqrt{1-\mathbb{E}_{1}} & 0 \\
0 & 0 & 0 & -e^{i \delta}
\end{array}\right)
$$

where $\mathbb{E}_{1} \ll 1$ is the nonadiabatic switching probability between states $|01\rangle$ and $|10\rangle, \phi$ is an arbitrary phase angle, and $\delta \ll 1$ is a controlled-phase error angle. Because $\delta$ is an angle, the leading-order error associated with it is of order $\delta^{2}$. The component of (29) in the $\{|01\rangle,|10\rangle\}$ subspace is assumed to be exactly unitary. The origins of these errors are discussed in Ref. [24]. Note that (29) does not include leakage out of the computational basis. It is useful to relate the size of the errors $\mathbb{E}_{1}$ and $\delta$ to the state-averaged gate fidelity [25, 26]

$$
F_{\text {ave }}\left(U, U_{\text {target }}\right) \equiv \frac{\operatorname{Tr}\left(U^{\dagger} U\right)+\left|\operatorname{Tr}\left(U_{\text {target }}^{\dagger} U\right)\right|^{2}}{20},
$$

where $U$ is the realized time-evolution operator (29) and $U_{\text {target }}=\mathrm{CZ}$. We find that the intrinsic gate error

$$
\mathbb{E} \equiv 1-F_{\text {ave }}
$$

is, to leading order,

$$
\mathbb{E}=\frac{2}{5} \mathbb{E}_{1}+\frac{3}{20} \delta^{2}
$$

To map $U$ to a Pauli channel we write it as

$$
U=V \times \mathrm{CZ}
$$

where

$$
V=\left(\begin{array}{cccc}
1 & 0 & 0 & 0 \\
0 & \sqrt{1-\mathbb{E}_{1}} & \sqrt{\mathbb{E}_{1}} e^{i \phi} & 0 \\
0 & -\sqrt{\mathbb{E}_{1}} e^{-i \phi} & \sqrt{1-\mathbb{E}_{1}} & 0 \\
0 & 0 & 0 & e^{i \delta}
\end{array}\right)
$$

is the error. Expanding (34) in the two-qubit Pauli basis $\mathcal{A}_{2}$ leads to

$$
\begin{aligned}
V & =\frac{1+2 \sqrt{1-\mathbb{E}_{1}}+e^{i \delta}}{4} I+\frac{1-e^{i \delta}}{4}\left(Z_{1}+Z_{2}\right) \\
& +\frac{i \sqrt{\mathbb{E}_{1}} \sin \phi}{2}(X X+Y Y)-\frac{i \sqrt{\mathbb{E}_{1}} \cos \phi}{2}(X Y-Y X) \\
& +\frac{1-2 \sqrt{1-\mathbb{E}_{1}}+e^{i \delta}}{4} Z Z
\end{aligned}
$$

In the PTA we therefore model the CZ gate (29) by the ideal gate (28) followed by the application of the twoqubit Pauli error channel

$$
\begin{aligned}
\rho & \rightarrow p_{I} \rho+p_{Z_{1}} Z_{1} \rho Z_{1}+p_{Z_{2}} Z_{2} \rho Z_{2}+p_{X X} X X \rho X X \\
& +p_{Y Y} Y Y \rho Y Y+p_{X Y} X Y \rho X Y+p_{Y X} Y X \rho Y X \\
& +p_{Z Z} Z Z \rho Z Z
\end{aligned}
$$

where

$$
\begin{aligned}
& p_{I}=\left|\frac{1+2 \sqrt{1-\mathbb{E}_{1}}+e^{i \delta}}{4}\right|^{2} \approx 1-\frac{\mathbb{E}_{1}}{2}-\frac{3}{16} \delta^{2}, \\
& p_{Z_{1}}=p_{Z_{2}}=\left|\frac{1-e^{i \delta}}{4}\right|^{2} \approx \frac{\delta^{2}}{16}, \\
& p_{X X}=p_{Y Y}=\left|\frac{\sqrt{\mathbb{E}_{1}} \sin \phi}{2}\right|^{2}=\frac{\sin ^{2} \phi}{4} \mathbb{E}_{1} \leq \frac{\mathbb{E}_{1}}{4}, \\
& p_{X Y}=p_{Y X}=\left|\frac{\sqrt{\mathbb{E}_{1}} \cos \phi}{2}\right|^{2}=\frac{\cos ^{2} \phi}{4} \mathbb{E}_{1} \leq \frac{\mathbb{E}_{1}}{4}, \\
& p_{Z Z}=\left|\frac{1-2 \sqrt{1-\mathbb{E}_{1}}+e^{i \delta}}{4}\right|^{2} \approx \frac{\delta^{2}}{16} .
\end{aligned}
$$

\section{BELL STATE PRESERVATION}

In this work we do not simulate an actual encoded qubit, but rather a system having a similar (but smaller) stabilizer-measurement circuit. The physical layout of the system we study is shown in Fig. 1] This arrangement shows that the four-qubit system can be regarded as a small section of surface code [27 29]. However, because the number of data qubits is equal to the number of measured stabilizer generators, no logical qubits are encoded.

\section{A. Error correction protocol}

Two data qubits, 1 and 2, are prepared in a Bell state

$$
\left|B_{1}\right\rangle \equiv \frac{|00\rangle+|11\rangle}{\sqrt{2}}
$$




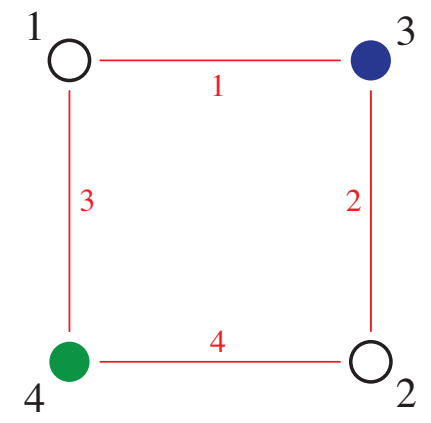

FIG. 1. (Color online) Physical layout of the four-qubit system, emphasizing its relation to the surface code. Open circles 1 and 2 are data qubits, the blue (dark gray) filled circle 3 is a $z$-type ancilla (syndrome) qubit, and the green (light gray) filled circle 4 is an $x$-type ancilla. The numbered lines indicate the presence of $\mathrm{CZ}$ gates and their sequence during each error-detection cycle.

and subjected to amplitude and phase damping. We try to preserve this state by repeatedly measuring the twoqubit stabilizer operator $Z Z$, using ancilla (syndrome) qubit 3 , and the operator $X X$ using a second ancilla qubit 4. Ideal simultaneous measurement of these commuting operators projects the data qubits into one of the four Bell states shown in Table

TABLE II. Simultaneous eigenfunctions of the stabilizer generators $X X$ and $Z Z$ with the corresponding ancilla qubit readouts $x_{3}$ and $x_{4}$.

\begin{tabular}{|c|cc|cc|}
\hline stabilizer state & $Z Z$ eigenvalue & $X X$ eigenvalue & $x_{3}$ & $x_{4}$ \\
\hline$B_{1}=2^{-\frac{1}{2}}(|00\rangle+|11\rangle)$ & 1 & 1 & 0 & 0 \\
$B_{2}=2^{-\frac{1}{2}}(|00\rangle-|11\rangle)$ & 1 & -1 & 0 & 1 \\
$B_{3}=2^{-\frac{1}{2}}(|01\rangle+|10\rangle)$ & -1 & 1 & 1 & 0 \\
$B_{4}=2^{-\frac{1}{2}}(|01\rangle-|10\rangle)$ & -1 & -1 & 1 & 1 \\
\hline
\end{tabular}

The Bell state preservation protocol we simulate is shown in Fig. 2. After preparing the data qubits in the state (38), a series of error-detection cycles are performed. Each cycle consists of preparing the ancilla qubits in the state $|0\rangle$, and performing an ancilla-assisted measurement of $Z Z$ and $X X$ with the gates shown. Here $\mathrm{H}$ is the Hadamard gate and the vertical lines are $\mathrm{CZ}$ gates. We have rewritten the measurement circuit in terms of the CZ gate (instead of the CNOT gate) for convenience of application to superconducting qubits [24]. The ancilla qubits are then measured in the diagonal basis, and the results $\left(x_{3}, x_{4}\right)$ recorded. The cycle is repeated, without measuring or resetting the data qubits.

In the absence of any errors, $\left(x_{3}, x_{4}\right)$ will be equal to $(0,0)$ cycle after cycle. However decoherence and gate errors lead to errors on the data qubits, ancilla qubits, or both. In the low-error-rate limit, data qubit errors are identified by a change of syndrome values from $(0,0)$ to $(0,1),(1,0)$, or $(1,1)$. The new values of $\left(x_{3}, x_{4}\right)$ will be observed until another error occurs. In this situation

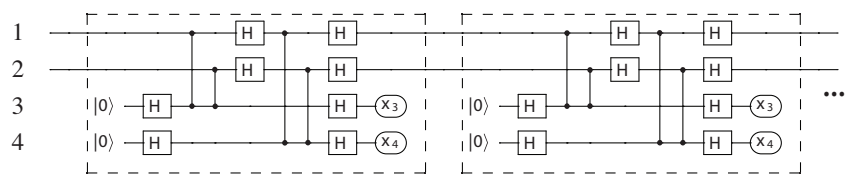

FIG. 2. Quantum circuit for the Bell state preservation protocol. Data qubits 1 and 2 are initialized to state (38). The stabilizer measurement cycle (dashed box) is repeated until the ancilla qubits 3 and 4 return identical values $\left(x_{3}, x_{4}\right)$ upon measurement for three consecutive cycles. The resulting observed values $\left(x_{3}, x_{4}\right)$ are used to predict the state of the data qubits, which are then measured in the Bell basis.

we can use Table $\amalg$ to predict the new state of the data qubits. Although we cannot uniquely identify the physical error leading to an observed syndrome change (for example, single-qubit errors $Z_{1}$ and $Z_{2}$ both change $\left|B_{1}\right\rangle$ to $\left.\left|B_{2}\right\rangle\right)$, it is still possible to correct the error-apply an operation to return to $\left|B_{1}\right\rangle$-if desired.

Errors can also occur on the ancilla qubits. For example, if the data qubits are in the state $\left|B_{1}\right\rangle$ but a bit-flip error $X_{4}$ occurs on qubit 4 immediately before readout, then syndrome values $(0,1)$ will be observed, but it will be incorrect to conclude that the data qubits are in state $\left|B_{2}\right\rangle$. However, unless the errors are correlated in time, the syndrome values will return to $(0,0)$ the next cycle. Thus, to protect against ancilla qubit errors it is necessary to run several measurement cycles and ignore single-cycle syndrome changes.

These observations motivate the following Bell state preservation protocol: In each trial, which represents a single experimental run, the data qubits are initially prepared in state (38), and the error-detection cycle is repeated until three consecutive ancilla measurements yield the same syndrome values

$$
\left(x_{3}, x_{4}\right)_{\text {final }} \text {. }
$$

Note that (39) is not necessarily equal to $(0,0)$ because data qubit errors may have occured before the stable values are reached. At this stage one could use (39) to predict the state of the data and hence the operation required to restore (38) - thereby "preserving" that initial Bell state - but instead we simply compare that prediction with a measurement of the data qubits in the Bell basis. This measurement verifies the preservation and completes the trail. It occurs after the final syndrome measurement and is the only time the data qubits are measured during the trial. The success probability for this trial is given by the probability $p_{B}$ of observing the predicted Bell state $|B\rangle$ upon measurement.

In what follows, we will be interested in the errorcorrection failure probability

$$
P \equiv \overline{1-p_{B}}
$$

averaged over many trials, in the presence of decoherence and intrinsic gate errors. The failure probability (40) is similar to a logical error rate, and we calculate $P$ exactly - by direct simulation - and by using the PTA. 


\section{B. Simulation results}

There are three natural approaches to actually carrying out the PTA simulations: The first is to generate the Pauli errors stochastically and track their effects using stabilizer group techniques [11]; this is the standard classically efficient approach used to calculate logical error rates and fault-tolerant thresholds. The second is to generate the Pauli errors stochastically, but track their effects through a full density matrix simulation; this (inefficient) option is simpler to implement but still requires a careful analysis of sampling errors. In particular, it is important to make sure that the error bars due to sampling are smaller than any changes of or differences in $P$ of interest. The third approach, which we follow here, is to fold the PTA-derived Pauli channels back into Kraus maps, which are then implemented exactly. This is also inefficient but avoids the introduction of Monte Carlo sampling errors.

We have performed a variety of simulations and find that the PTA is remarkably accurate. Representative results are shown in Fig. 3. Here the error-correction failure rate (40) is plotted as a function of the decoherence rate, for a wide range of intrinsic gate errors (31). The decoherence rate in Fig. 3 is changed by varying $T_{1}$ with $T_{2}=T_{1}$ (and noise-spectrum parameter $\alpha=0$ ), and is plotted, not as a function of $T_{1}$ itself, but as a function of the total probability

$$
p_{\text {step }} \equiv p_{X}+p_{Y}+p_{Z}
$$

of a decoherence-induced error per step, where the $p_{a}$ are the single-step Pauli error probabilities (20). Note that in Fig. 3 (and Figs. 4 and 5, which also have $T_{2}=T_{1}$ ),

$$
p_{\text {step }} \approx \frac{3 t_{\text {step }}}{4 T_{1}} .
$$

The single-step operation time is taken to be

$$
t_{\text {step }}=25 \mathrm{~ns} .
$$

Because there are nine steps per cycle in the measurement circuit of Fig. 2, this value of $t_{\text {step }}$ results in an error-correction cycle time of $225 \mathrm{~ns}$, a value appropriate for superconducting architectures [29].

The nonideal CZ gate (29) is characterized by the three parameters $\mathbb{E}_{1}, \delta$, and $\phi$. As discussed in Sec. IIB, $\mathbb{E}_{1}$ is the nonadiabatic transition probability between the $|01\rangle$ and $|10\rangle$ states, $\phi$ is the associated phase angle, and $\delta$ is a controlled-phase error angle. In Fig. 3 we assume that $\phi=0$. We do find a small degree of sensitivity to this phase: For example, changing $\phi$ to $\frac{\pi}{4}$ (or $\frac{\pi}{2}$ ) leads

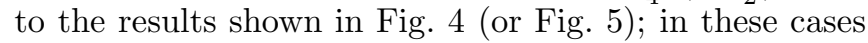
the PTA accuracy is slightly worse. Note from (37) that the value of $\phi$ determines the strength of two-qubit Pauli errors of the form $X X, X Y, Y X$, and $Y Y$, and that in Fig. 4, the PTA always overestimates $P$ but in Fig. $[5$ it underestimates the case $\mathbb{E}_{1}=10 \%$ but overestimates the

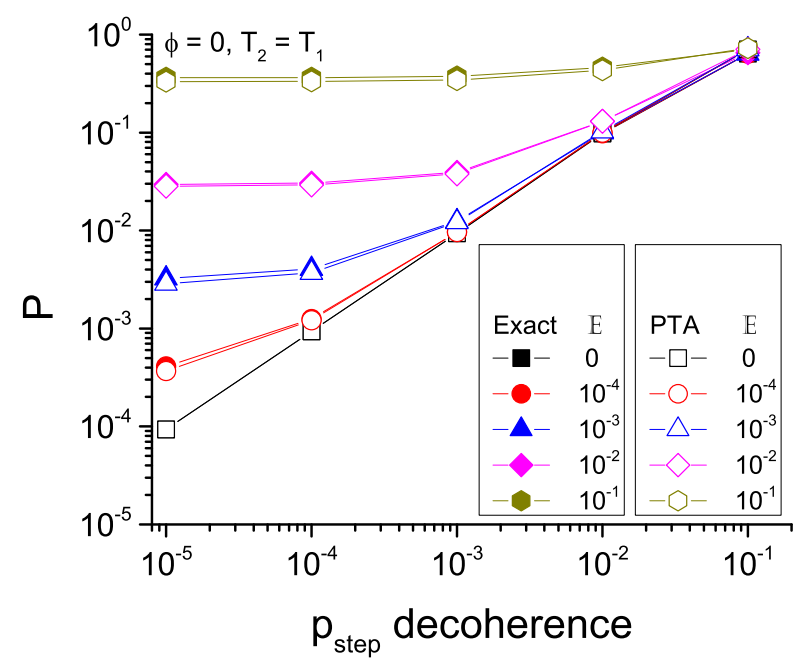

FIG. 3. (color online) Error-correction failure probability (40) versus per-step decoherence rate, assuming $T_{2}=T_{1}$. Here $p_{\text {step }}$ is the total probability (41) of a decoherence-induced error per $25 \mathrm{~ns}$ step of the circuit of Fig. 2. The case where there is decoherence present but no gate errors is shown in black; the agreement is essentially perfect. In addition, $\mathbb{E}$ is the total intrinsic gate error (31) equally distributed over the two error mechanisms (characterized by $\mathbb{E}_{1}$ and $\delta$ ) present in the nonideal $C Z$ gate (29). The explicit values of $\mathbb{E}_{1}$ and $\delta$ used are provided in (44). The PTA values (open symbols) are hardly distinguishable from the exact results (filled symbols).

others. The values of $\mathbb{E}_{1}$ and $\delta$ simulated are such that they each lead to one half of the total intrinsic gate error (31). Therefore we use

$$
\mathbb{E}_{1}=\frac{5 \mathbb{E}}{4} \quad \text { and } \quad \delta=\sqrt{\frac{10 \mathbb{E}}{3}}
$$

which follows from (32).

In Figs. 3 and 4 we assume $T_{2}=T_{1}$. This means that the PTA maps the decoherence process to the depolarizing channel with $p_{X}=p_{Y}=p_{Z}$. In Fig. [6 we show results as in Fig. 3 but with $T_{2}=2 T_{1}$, and in Fig. 7 we show results for $T_{2}=\frac{1}{2} T_{1}$. Both show excellent performance of the PTA.

\section{CONCLUSIONS AND POSSIBLE DIRECTIONS FOR FUTURE WORK}

Although we did not calculate the actual logical error rate associated with an encoded qubit, our results suggest that the performance of the PTA will be similar in that case for small topological codes. In fact the performance found here is sufficiently good that there is probably little room for further improvement in small codes. However, it must be emphasized that the accuracy of the PTA may not extend to large-distance codes, 


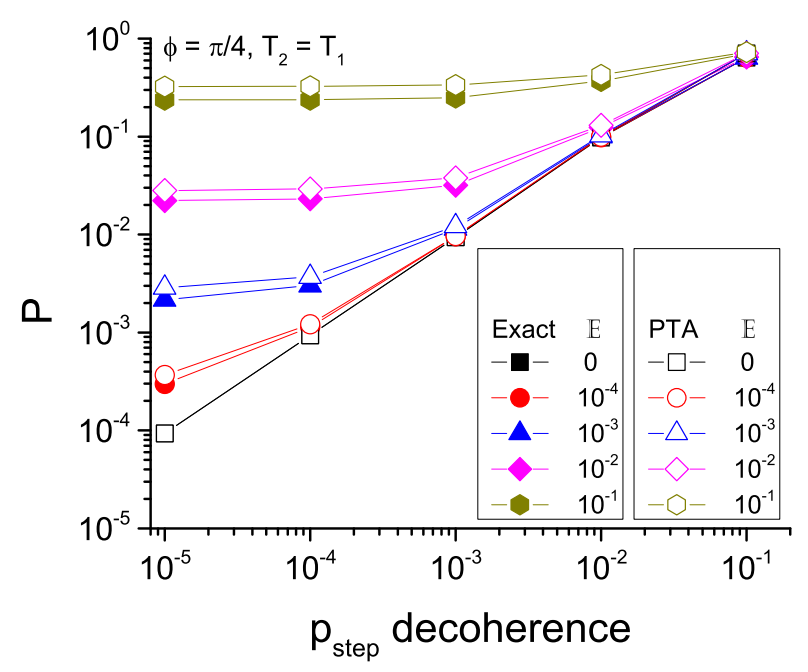

FIG. 4. (color online) Same as Fig. 3 but with $\phi=\frac{\pi}{4}$.

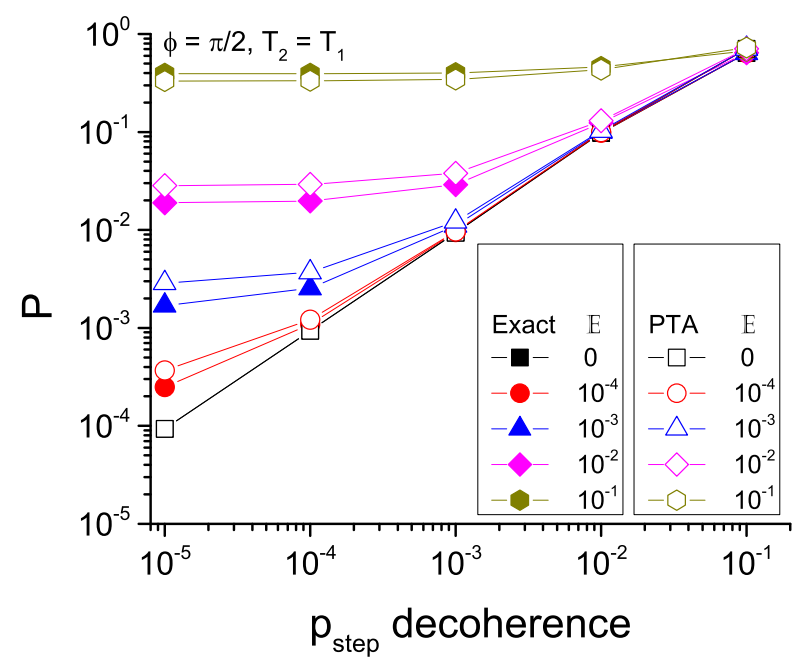

FIG. 5. (color online) Same as Fig. 3 but with $\phi=\frac{\pi}{2}$.

and this remains an important open question. It would be interesting to go beyond the full Hilbert space simulations reported here and develop analytical or numerical approximation methods, based on more conventional many-body theory techniques, to calculate the logical error rates in large-distance codes for comparison with the PTA and other efficient error models.

It would also be useful to understand why the PTA works so well. For example, it is known that the "offdiagonal" terms dropped in the PTA are upper-bounded by the diagonal PTA probabilities $p_{A}$ [19]. Perhaps this bound on their magnitude, combined with the fact that these terms also come with phase factors that allow for

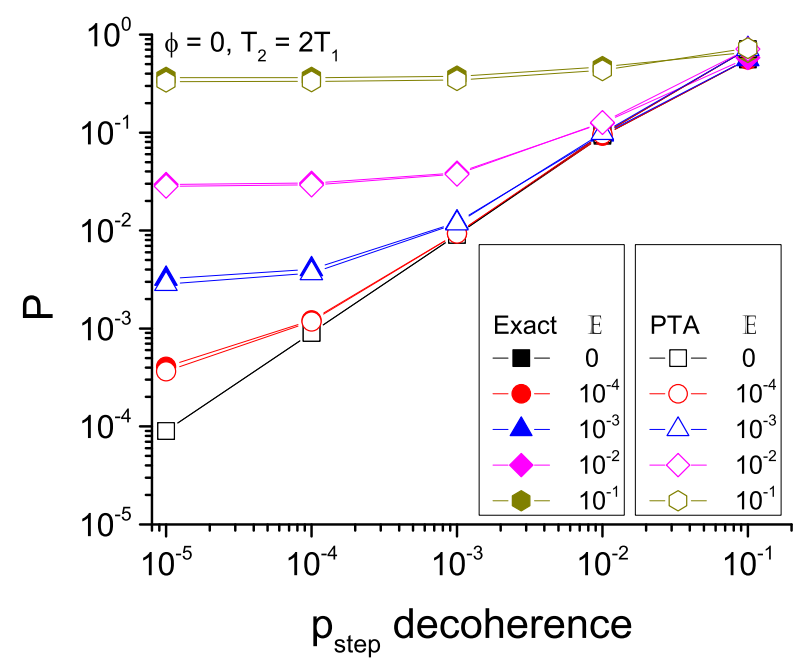

FIG. 6. (color online) Error-correction failure probability with $T_{2}=2 T_{1}$. The values of $\mathbb{E}_{1}$ and $\delta$ are determined from Eq. (44).

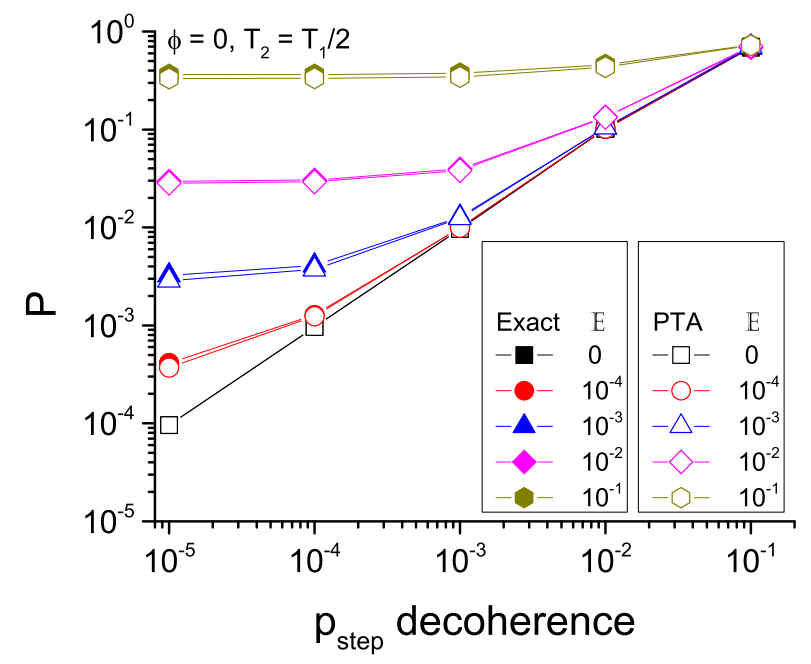

FIG. 7. (color online) Error-correction failure probability with $T_{2}=\frac{1}{2} T_{1}$. The values of $\mathbb{E}_{1}$ and $\delta$ are determined by (44).

partial cancellation, can explain our observation.

The PTA does not necessarily upper-bound the actual error. It does in some examples but does not in others. However the violation is negligible in the small system studied here, and in our opinion the accuracy of the approximation is more important than the bounding property. In addition, we have shown that the PTA can be simply modified to make it upper-bound the errorcorrection failure probability $P$ : The modification is to 
take the largest PTA-derived probability

$$
p_{0} \equiv \max _{A}\left\{p_{A}\right\}
$$

for the process in question and construct a new Pauli channel where all (non-trivial) errors occur with probability $p_{0}$. But the accuracy of the resulting approximation is somewhat compromised.

The combined simplicity and accuracy of the PTA suggests that, at a minimum, it be used as a starting point for further refinements. For example, it might be possible to develop an efficient protocol to perturbatively include the leading order corrections to the PTA, i.e., the leadingorder effects of the neglected off-diagonal terms. Such an approach might also allow one to assess the range of validity of the PTA and whether is breaks down in large codes

\section{ACKNOWLEDGMENTS}

This research was funded by the US Office of the Director of National Intelligence (ODNI), Intelligence Advanced Research Projects Activity (IARPA), through the US Army Research Office grant No. W911NF-10-1-0334. All statements of fact, opinion or conclusions contained herein are those of the authors and should not be construed as representing the official views or policies of IARPA, the ODNI, or the US Government.

It is a pleasure to thank Austin Fowler, Joydip Ghosh, and John Martinis for useful discussions. Part of this work was carried out while M.G. was a Lady Davis Visiting Professor in the Racah Institute of Physics at Hebrew University, Jerusalem.
[1] D. Aharonov and M. Ben-Or, in Proceedings of the 29th Annual IEEE Symposyum on the Theory of Computer Science (ACM, 1997) pp. 176-88.

[2] A. Kitaev, Russ. Math. Surv., 52, 1191 (1997).

[3] E. Knill, R. Laflamme, and W. H. Zurek, Proc. Roy. Soc. Lond. A, 454, 356 (1998).

[4] D. Aharonov and M. Ben-Or, SIAM J. COMPUT., 38, 1207 (2008).

[5] A. G. Fowler, Phys. Rev. Lett., 109, 180502 (2012).

[6] B. M. Terhal and G. Burkard, Phys. Rev. A, 71, 012336 (2005).

[7] P. Aliferis, D. Gottesman, and J. Preskill, Quant. Info. and Comp., 6, 97 (2006), quantph/0504218.

[8] D. Aharonov, A. Kitaev, and J. Preskill, Phys. Rev. Lett., 96, 050504 (2006).

[9] E. Novais, E. R. Mucciolo, and H. U. Baranger, Phys. Rev. A, 012314 (2008).

[10] E. Novais and E. R. Mucciolo, Phys. Rev. Lett., 110, 010502 (2013).

[11] D. Gottesman, "Stabilizer codes and quantum error correction," (1997), PhD thesis, California Institute of Technology. arXiv:quant-ph/9705052.

[12] S. Aaronson and D. Gottesman, Phys. Rev. A, 70, 052328 (2004).

[13] E. Magesan, D. Puzzuoli, C. E. Granade, and D. G. Cory, Phys. Rev. A, 87, 012324 (2013).

[14] M. Gutiérrez, L. Svec, A. Vargo, and K. R. Brown, Phys. Rev. A, 87, 030302 (2013).

[15] C. H. Bennett, D. P. DiVincenzo, J. A. Smolin, and W. K. Wootters, Phys. Rev. A, 54 (1996).

[16] W. Dür, M. Hein, J. I. Cirac, and H.-J. Briegel, Phys.
Rev. A, 72, 052326 (2005).

[17] J. Emerson, M. Silva, O. Moussa, C. Ryan, M. Laforest, J. Baugh, D. G. Cory, and R. Laflamme, Science, 317, 1893 (2007).

[18] C. Dankert, R. Cleve, J. Emerson, and E. Livine, Phys. Rev. A, 80, 012304 (2009).

[19] C. C. Lopez, A. Bendersky, J. P. Paz, and D. G. Cory, Phys. Rev. A, 81, 062113 (2010).

[20] A. G. Fowler, A. C. Whiteside, A. L. McInnes, and A. Rabbani, Phys. Rev. X, 2, 041003 (2012).

[21] P. K. Sarvepalli, A. Klappenecker, and M. Rotteler, Proc. Roy. Soc. A, 465, 1645 (2009).

[22] J. Ghosh, A. G. Fowler, and M. R. Geller, Phys. Rev. A, 86, 062318 (2012).

[23] F. W. Strauch, P. R. Johnson, A. J. Dragt, C. J. Lobb, J. R. Anderson, and F. C. Wellstood, Phys. Rev. Lett., 91, 167005 (2003).

[24] J. Ghosh, A. Galiautdinov, Z. Zhou, A. N. Korotkov, J. M. Martinis, and M. R. Geller, Phys. Rev. A, 87, 022309 (2013).

[25] P. Zanardi and D. A. Lidar, Phys. Rev. A, 70, 012315 (2004).

[26] L. H. Pedersen, N. M. Moller, and K. Molmer, Phys. Lett. A, 367, 47 (2007).

[27] S. B. Bravyi and A. Y. Kitaev, "Quantum codes on a lattice with boundary," (1998), arXiv:9811052.

[28] R. Raussendorf and J. Harrington, Phys. Rev. Lett., 98, 190504 (2007).

[29] A. G. Fowler, M. Mariantoni, J. M. Martinis, and A. N. Cleland, Phys. Rev. A, 86, 032324 (2012). 